\title{
Consecuencias en el congelamiento en el beneficiado de Vanilla planifolia Andrews
}

Consequences in the freezing in the beneficiary of Vanilla planifolia Andrews

\author{
Ramírez Juárez Sharon ${ }^{1 凶}$, García Muñoz Silvia Amanda², Ortega Rodríguez Anabel² Villareal Ramírez Víctor \\ H. ${ }^{2}$, Leyva Chavez Arwell Nathan ${ }^{2}$, Uranga Valencia Luisa P. ${ }^{2}$ \\ ${ }^{1}$ Facultad de Ciencias Biológico Agropecuarias, Universidad Veracruzana. ${ }^{2}$ Facultad de Ciencias \\ Agrotecnológicas, Universidad Autónoma de Chihuahua. \\ ${ }^{\bowtie}$ Autor para correspondencia: sharon ri23@hotmail.com
}

Recibido: $15 / 04 / 2019$

Aceptado: 15/05/2019

\section{RESUMEN}

El objetivo principal del experimento fue determinar el efecto del congelamiento en el beneficio de vainilla de pequeños productores de la sierra de Otontepec, Veracruz. Los tratamientos utilizados fueron vainas congeladas durante seis meses y un año y medio, recolectadas en cinco sitios: Estación, Tamalinillo, Xochitlán, Tancoco y Agua Salada. Las variables de respuesta utilizadas fueron contenidas de vainillina, tamaño y peso de las vainas. Para determinar el contenido de vainillina de cada tratamiento se utilizó el método por el análisis de Cromatografía Liquida de alta Resolución (HPLC), los resultados mostraron que el efecto del congelamiento no afectó la concentración de vainillina a pesar del tiempo en que las vainas fueron congeladas, obteniendo concentraciones altas que va desde 3.19\% - 14.33\%. Así mismo se observó que el tamaño y el peso de las vainas no tienen influencia en el contenido de vainillina, obteniendo tamaño promedio entre $15 \mathrm{~cm}-19.26 \mathrm{~cm}$, catalogándose tres de los cinco sitios muestreados con vainas de calidad extra, y un peso promedio de entre $1.48 \mathrm{~g}-3.79 \mathrm{~g}$.

Palabras clave: Caidad, HPLC, Vainillina, Alteración.

\begin{abstract}
The main objective of the experiment was to determine the effect of freezing on the vanilla benefit of small producers in the Sierra de Otontepec, Veracruz. The treatments used were frozen pods for six months and a year and a half, collected in five sites: Estación, Tamalinillo, Xochitlán, Tancoco and Agua Salada. The response variables used were vanillin content, pod size and weight. To determine the vanillin content of each treatment the method was used by the High Resolution Liquid Chromatography (HPLC) analysis, the results showed that the effect of freezing did not affect the vanillin concentration despite the time in which the pods were frozen, obtaining high concentrations ranging from $3.19 \%-14.33 \%$. It was also observed that the size and weight of the pods have no influence on the vanillin content, obtaining an average size between $15 \mathrm{~cm}-19.26 \mathrm{~cm}$, listing three of the five sites sampled with extra quality pods, and an average weight of between $1.48 \mathrm{~g}-3.79 \mathrm{~g}$.
\end{abstract}

Keywords: Quality, HPLC, Vanillin, Alteration. 


\section{INTRODUCCIÓN}

La vainilla es considerada como una aportación de México para el mundo. Esta orquídea es considerada como una planta que se cultiva para cosechar sus vainas, de las cuales se puede obtener un valioso extracto (vegetal) y que es utilizado para agregar sabor y aroma a diversos alimentos y bebidas, así como en productos de farmacia, cosméticos, tabaco, y artesanías (SAGARPA, 2016).

En México se ha llevado a cabo el proceso de curado o beneficiado, permitiendo potencializar y acelerar el proceso natural en el desarrollo de sabor y aroma característico de la vainilla. Los beneficiadores mexicanos aplican diferentes procesos que definen de acuerdo a las condiciones climáticas, disponibilidad del producto, destino o uso de la vainilla, o si realizan el beneficio por el método tradicional (bajo sol) o tecnificado (horno) (Jaramillo et al., 2013). Aunque ambos sistemas son diferentes tienen en común cuatro etapas principales: marchitado, sudado, secado y acondicionado (Ramachandra-Rao y Ravishankar, 2000). Sin embargo, dentro de cada uno de estos pasos existen variantes dependiendo de cambios en el clima donde se ubica el beneficiado, madurez de los frutos, volumen de los mismos a beneficiar, así como, disponibilidad y experiencia en la mano de obra (Odoux, 2011). Cada beneficiador se enfrenta a diferentes problemas y como resultado de la interacción de esos factores se presenta gran variabilidad en la calidad de los frutos beneficiados, que impacta significativamente en su perfil de aroma, y que puede o no satisfacer los estándares nacionales e internacionales.

En la actualidad, se han desarrollado varias investigaciones para determinar las condiciones óptimas, en que se obtiene una buena calidad de la vainilla curada o beneficiada (Dignum et al.,
2002). Sin embargo, el beneficiado mexicano es considerado de gran calidad internacionalmente. Con el fin de conservar las características optimas de la vainilla, en esta investigación de determinaran las consecuencias en el congelamiento en el beneficiado de Vanilla planifolia Andrews, evaluando contenido de vainillina, tamaño y peso de las vainas de cinco beneficios de vainilla ubicados en la Sierra de Otontepec, Veracruz.

\section{MATERIALES Y MÉTODOS}

A las vainas utilizadas para este análisis, se les realizó una maceración de aproximadamente 48 horas, pesando 1 gramo de vaina por cada muestra, colocando cada una en frascos ámbar y agregándole a cada frasco 20 mililitros de alcohol etílico. Transcurrido el tiempo de maceración se micro filtraron las muestras utilizando micro filtros de $0.20 \mu \mathrm{m}$ (micrómetros) y cada muestra se colocó en frascos de vidrio previamente esterilizados.

Cromatógrafo de líquidos (HPLC por sus siglas) Para la utilización del cromatógrafo se empleó el programa Galaxie Chromatotography Data System, una vez iniciado el programa, se colocó la fase móvil de metanol para realizar una purga con una duración de $30 \mathrm{~min}$.

Posteriormente se realizó una corrida de $10 \mathrm{~min}$. Para estabilizar el equipo de cromatografía liquida de alta resolución HPLC, con una longitud de onda de $254 \mathrm{~nm}$ y con una presión de 270.

Para realizar las determinaciones de vainillina se realizaron corridas de 5 minutos cada una, con una longitud de onda de $254 \mathrm{~nm}$, inyectando 20 $\mu 1$ por muestra, con una aguja de inyección especial para cromatografía. 


\section{RESULTADOS Y DISCUSIÓN}

Las muestras de vainas fueron recolectadas en cinco sitios pertenecientes al área natural protegida de la Sierra Otontepec, Veracruz. De las cuales cuatro sitios realizaron el beneficiado por congelamiento en vainas durante seis meses, mientras que un sitio realizo el beneficiado en un año y seis meses, contando con diferentes tipos de tutor, como se muestra a continuación:

Cuadro 1. Asociación de tutores de vainilla

\begin{tabular}{|c|c|c|}
\hline SITIO & DURACION DE CONGELAMIENTO & TUTOR \\
\hline Estación & Un año y medio & Espalderas + malla sombra \\
\hline Tamalinillo & Seis meses & Citrus aurantifolia \\
\hline Xochitlan & Seis meses & Erythrina \\
\hline Tancoco & Seis meses & Bursera simaruba + citrus \\
\hline Agua salada & Seis meses & Citrus sinensis \\
\hline
\end{tabular}

Contenido de vainillina por Cromatografía liquida de alta resolución (HPLC).

Para esta variable de respuesta los resultados obtenidos se cuantificaron individualmente dependiendo del sitio de cada muestra y en función de las respectivas áreas que cada pico registró a una longitud de onda de $254 \mathrm{~nm}$ como se muestra en el cuadro 2 .

Cuadro 2. Concentración vainillina.

\begin{tabular}{|c|c|c|c|}
\hline MUESTRA & $\begin{array}{c}\text { TIEMPO } \\
\text { (min.) }\end{array}$ & $\begin{array}{c}\text { ÁREA } \\
\text { (longitud de } \\
\text { onda) }\end{array}$ & $\begin{array}{c}\text { PORCENTAJE } \\
(\%)\end{array}$ \\
\hline Agua Salada & 4.41 & 20550.3 & 4.40 \\
\hline Estación & 4.35 & 66893.7 & 5.33 \\
\hline Tancoco & 4.35 & 18756.8 & 4.02 \\
\hline Xochitlán & 4.41 & 91456.3 & 6.59 \\
\hline Tamalinillo & 4.41 & 14879.6 & 3.19 \\
\hline
\end{tabular}

Los contenidos de vainillina que se registraron en las muestras analizadas fueron alrededor de $3.19 \%$ a $6.59 \%$, de los sitios Tamalinillo y Xochitlán respectivamente, estos valores reflejan contenidos arriba de lo normal superando los reportados por Waliszewski (2007) donde registró un máximo de contenido en vainillina de entre 6-7\%. Por otro lado, los sitios que obtuvieron concentraciones de entre $3.19 \%$ a $4.40 \%$ se encuentran dentro de los resultados reportados en otras regiones del mundo 3.4\% (Tapia et al., 2008).

Se coincide con Tapia et al., (2008) quienes suponen que la transformación de los precursores de vainillina durante su beneficiado no es eficiente y que la variación de las condiciones ambientales durante el crecimiento de las plantas repercute en el desarrollo de los componentes aromáticos de la vainilla. 


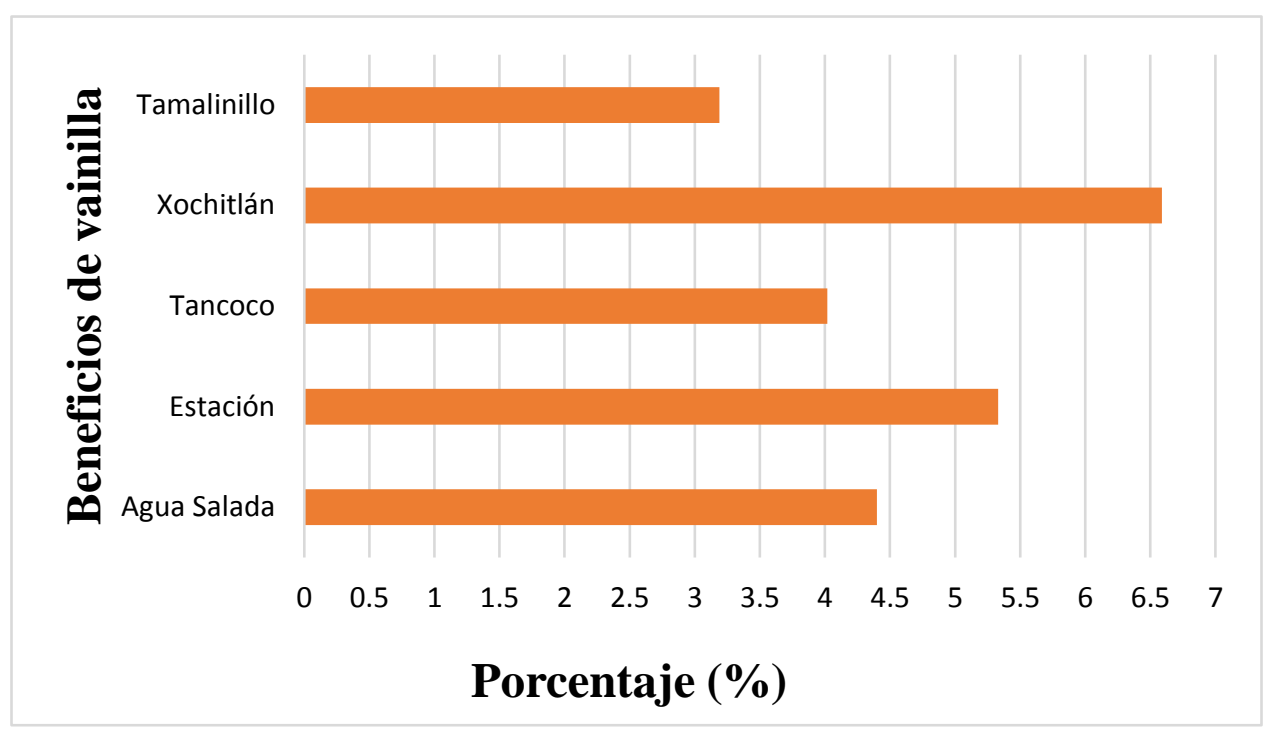

Figura 1. Porcentaje de concentración vainillina.

De acuerdo con el método realizado por Cromatografía liquida de Alta Resolución (HPLC), se puede observar que la duración del congelamiento no afectó los contenidos de vainillina, puesto que las vainas con un año y seis meses en congelación obtuvieron uno de los mayores contenidos de vainillina, las vainas pueden congelarse inclusive en menos de seis meses, existen otros métodos en donde congelan las vainas a temperaturas más severas en menos tiempo, sin embargo para los productores de vainilla de la sierra es más práctico congelarlo a una temperatura menos severa $\left(-4{ }^{\circ} \mathrm{C}\right)$ en refrigeradores que tienen a su disposición; lo cual concuerda con Ansaldi et al., (1990) quienes argumentan que el sabor natural de la vainilla mejora congelando las vainas entre $-5^{\circ}$ C Y $-30^{\circ} \mathrm{C}$; así como también, con Balls et al., (1941) que mencionan que al descongelar las vainas estas se caracterizan por tener mayor flexibilidad, color y aroma deseable, mejorando la calidad de la vainilla.

Tamaño de las vainas. El Cuadro 3 muestra los valores de tamaño de vainas de acuerdo con la NMX-FF-074-SCFI-2009 de acuerdo con la longitud promedio de esta clasificación, de las cinco muestras evaluadas, tres están dentro de la categoría extra: Estación $(19.26 \mathrm{~cm})$, Tamalinillo $(19.06 \mathrm{~cm})$, Tancoco $(18.94 \mathrm{~cm})$, mientras que Agua Salada $(16.3 \mathrm{~cm})$ y Xochitlán $(15 \mathrm{~cm})$ se encuentran como categoría primera.

Cuadro 3. Tamaño de la vaina en función de su longitud.

\begin{tabular}{|c|c|c|c|}
\hline \multicolumn{2}{|c|}{ CÓDIGO DE TAMAÑ } & INTERVALO DE LONGITUD & LONGITUD PROMEDIO \\
\hline & $\mathrm{A}$ & $23.0-21.9$ & 22.4 \\
\hline Extra & $\mathrm{B}$ & $21.8-20.6$ & 21.2 \\
\hline & $\mathrm{C}$ & $20.5-19.3$ & 19.9 \\
\hline & $\mathrm{D}$ & $19.2-18.1$ & 18.6 \\
\hline Primera & $\mathrm{E}$ & $18.0-16.8$ & 17.4 \\
\hline & $\mathrm{F}$ & $16.7-15.1$ & 16.1 \\
\hline & $\mathrm{G}$ & $15.0-14.2$ & 14.8 \\
\hline Segunda & $\mathrm{H}$ & $14.1-10.0$ & 12.1 \\
\hline
\end{tabular}

Fuente: NMX-FF-074-SCFI-2009. 
El tamaño de las vainas que se evaluaron se muestra en el cuadro 4, se puede observar un rango de tamaño de las vainas entre $\operatorname{los} 15 \mathrm{~cm}$ $19.26 \mathrm{~cm}$ concordando con los resultados obtenidos por Luna et al., (2016) al obtener rangos similares (14.5 cm- 20.9). Basado en la norma, los códigos de tamaño varían de acuerdo con las longitudes de las vainas para cada sitio.

Cuadro 4. Tamaños evaluados en vainas de Vanilla planofolia A.

\begin{tabular}{|c|c|c|c|}
\hline $\begin{array}{c}\text { CÓDIGO DE } \\
\text { TAMAÑ }\end{array}$ & $\begin{array}{c}\text { INTERVALO DE } \\
\text { LONGITUD }\end{array}$ & $\begin{array}{c}\text { LONGITUD } \\
\text { PROMEDIO }\end{array}$ & SITIO \\
\hline D & $19.6-18.7$ & 19.26 & Estación \\
\hline D & $19.5-18.5$ & 19.06 & Tamalinillo \\
\hline G & $15.1-15.5$ & 15 & Xochitlán \\
\hline D & $19.5-18.2$ & 18.94 & Tancoco \\
\hline F & $15.8-16.1$ & 16.13 & Agua Salada \\
\hline
\end{tabular}

El tamaño no afecta los contenidos de vainillina, sin embargo, es uno de los parámetros fisicoquímicos que influye en la calidad de la vainilla ya que el mercado demanda vainas con mayor tamaño, color y olor. Para que se puedan llegar a obtener vainas con buen tamaño es necesario contar con diferentes condiciones que favorezcan el crecimiento de las vainas como son: la cantidad de agua disponible, la cantidad de luz, nutrientes en el suelo, calidad de la tierra, temperatura, entre otras (Hernández-Hernández et al., 2011).

Peso de las vainas. Para esta variable de respuesta se obtuvo el peso promedio de cada sitio evaluado, como se muestra en el cuadro 6 obteniendo los promedios más altos en Tamalinillo (3.79 g), seguido de Tancoco (3.63 g), Estación (3.35 g), Agua Salada (2.15 g) y con el peso promedio más bajo Xochitlán (1.48 g).

Cuadro 5. Peso promedio de vainas.

\begin{tabular}{|c|c|}
\hline SITIO & PESO PROMEDIO \\
\hline Estación & $3.35 \mathrm{~g}$. \\
\hline Tamalinillo & $3.79 \mathrm{~g}$. \\
\hline Xochitlan & $1.48 \mathrm{~g}$. \\
\hline Tancoco & $3.63 \mathrm{~g}$. \\
\hline Agua Salada & $2.15 \mathrm{~g}$. \\
\hline
\end{tabular}

El peso de vainas no se encuentra dentro de los parámetros establecidos en la NMX-FF-074-
SCFI-2009, este no afecto contenido de vainillina, sin embargo, se cataloga como una de 
las características fisicoquímicas de la vainilla. Luna et al., (2016), realizó parámetros fisicoquímicos y sensoriales en vainilla, dentro de los parámetros evaluó tamaño y peso en vainas, sin embargo, solo mostró resultados en cuanto a tamaño para la clasificación de las vainas.

Los pesos encontrados en tres de los sitios de esta investigación, Tamalinillo $3.79 \mathrm{~g}$, Tancoco 3.63 g y Estación con 3.35 g, coinciden con lo reportado por eTD solutions (2007-2014) donde los pesos promedios de las vainas de procedencia mexicana se encuentran entre 3 y 6 gramos, así como también con el peso promedio de vainas procedentes de uno de los principales países productores, Madagascar, que obtienen rangos de 3 a 5 gramos.

\section{CONCLUSIÓN}

El método de Cromatografía líquida de alta resolución (HPLC) muestra que el efecto de la duración del congelamiento para el matado de vainas no afectó los contenidos de vainillina de los tratamientos evaluados, obteniendo concentraciones altas, de 3.19 a $19.59 \%$, tanto en vainas que se congelaron durante seis meses como las de un año y medio.

El congelamiento como método para el matado de vainas, es otra alternativa que los productores de la sierra Otontepec, Veracruz pueden adaptar fácilmente, ya que es más practico congelar las vainas que utilizar métodos como inmersiones que pueden afectar la calidad de la vainilla si no se realiza adecuadamente; se demostró que el tiempo de congelamiento no afecta el contenido de vainillina.

Con respecto a la longitud de las vainas, considerando la NMX-ff-074-SCFI-2009, tres de las muestras se catalogaron como categoría extra y las dos muestras restantes de primera categoría; obteniendo longitudes que van de los $15 \mathrm{~cm}$ a $19.26 \mathrm{~cm}$. En cuanto al peso, se encontraron los parámetros con un rango de 1.48 g a $3.79 \mathrm{~g}$. Las variables de tamaño y peso de las vainas no influyen en los contenidos de vainillina presentes en las vainas.

\section{LITERATURA CITADA}

Balls, A. K. y Arana, F.E. 1941. The curing of vanilla. Ind. Eng. Chem. 33, 1073-1075.https://doi.org/10.1021/ie503 $80 \mathrm{a} 026$

Dignum, M.J.W; Kerler, J; Verpoorte, R. 2002. Vanilla curing under laboratory conditions. Food Chem. 79: 165-171. https://doi.org/10.1016/S0308-8146(02)0 0125-5

Hernández-Hernández J. 2011. Mexican vanilla production. En: D. HavkinFrenkel, F.C. Belanger. Handbook of vanilla science and technology. Blackwell Publishing Co. West Sussex, UK. https://doi.org/10.1002/9781444329353. ch1

Jaramillo, V.J.L; Escobedo, G.J.S; Barrera, R.A; Herrera, C.B.E. 2013. Eficiencia económica en el beneficiado de vainilla (Vanilla planifolia J.) en la región del Totonacapán, México. Revista Mexicana de Ciencias Agrícolas. 4 (3): 477-483. https://doi.org/10.29312/remexca.v4i3.12 08

Luna, G.J.J; Luna, G.M.L; Amador, E.G.G. 2016. Caracterización fisicoquímica y sensorial de Vanilla planifolia jakcs ex. Andrews con diferentes esquemas de beneficiado. Agroproductividad, vol. 9, núm. 1, pp. 34-40.

Odoux, E. 2011. Vanilla Curing. En: Odoux E, Grisoni M (eds). Vanilla. (Medicinal and aromatic plants-industrial profiles). CRC Press. Boca Raton Florida. 173-185. https://doi.org/10.1201/EBK1420083378 
Ramachandra-Rao S. y Ravishankar G. A. 2000.

Vanilla flavour: Production by conventional and biotechnological routes. Journal of the Science of Food and Agriculture. 80: 189-304. https://doi.org/10.1002/1097-0010(20 0002)80:3<289::AID-JSFA543>3.0.C $\mathrm{O} ; 2-2$

SAGARPA. 2016. Vainilla mexicana. Planeación agrícola nacional 2017-2030. México.
Tapia, O. A. P; Dávila, O. G. 2008. Determinación de compuestos cromáticos de compuestos en vainilla mexicana (Vanilla planifolia Andrews) durante el beneficiado. V Congreso Internacional de Ingeniería Bioquímica. XVI congreso nacional de Ingeniería Bioquímica. VI Jornadas Científicas de Biomedicina y Biotecnología Molecular. México D.F.

Waliszewski K. N; Ovando S. L; Pardio, V. T. 2007. Effect of hydration and enzymatic petreatment of vanilla beans on the kinetics of vanillin extraction. Journal of Food Engineering. 78: 1267-1273. https://doi.org/10.1016/j.jfoodeng.2006.0 1.029

Copyright (c) 2019 Sharon Ramirez Juárez, Silvia Amanda G arcia Muñoz, Anabel Ortega Rodriguez, Victor H. Villarreal Ram irez, Arwell Nathan L eyva Chavez, Luisa P. Uranga V alencia

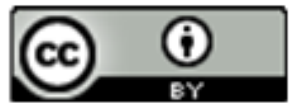

Este texto está protegido por una licencia licencia CreativeCommons 4.0.

Usted es libre para Compartir — copiar y redistribuir el $\mathrm{m}$ aterial en cualquier medio o formato-y Adaptar el documento —remezclar, transformar y crear a partir del material- para cualquier propósito, incluso para fines com erciales, siempre que cumpla la condición de:

Atribución: Usted debe dar crédito a la obra original de manera adecuada, proporcionar un enlace a la licencia, e in dicar si se han realizado cambios. Puede hacerlo en cualquier forma razonable, pero no de forma tal que sugiera que tiene el apoyo del licenciante o 10 recibe por el uso que hace de la obra.

Resumenclelicencia - Textocompletocklalicencia 